\title{
Tract-Based Spatial Statistics: Application to Mild Cognitive Impairment
}

\author{
Yau-Yau Wai, ${ }^{1,2}$ Wen-Chuin Hsu, ${ }^{3,4}$ Hon-Chung Fung, ${ }^{3}$ Jiann-Der Lee, ${ }^{5}$ Hsiao-Lung Chan, \\ Ming-Lun Tsai, ${ }^{2}$ Yu-Chun Lin, ${ }^{1,2}$ Yih-Ru Wu, ${ }^{3}$ Leslie Ying, ${ }^{6,7}$ and Jiun-Jie Wang ${ }^{1,2,8,9,10}$ \\ ${ }^{1}$ Department of Medical Imaging and Radiological Sciences, Chang Gung University, Taoyuan County, Taiwan \\ ${ }^{2}$ Department of Radiology and Intervention, Chang Gung Memorial Hospital, 5 FuHsing Street, Linkou, Taoyuan County, Taiwan \\ ${ }^{3}$ Department of Neurology, Chang Gung Memorial Hospital, Chang-Gung University College of Medicine, Linkou, \\ New Taipei 333, Taiwan \\ ${ }^{4}$ Dementia Center, Chang Gung Memorial Hospital, 5 FuHsing Street, Linkou, Taoyuan County, Taiwan \\ ${ }^{5}$ Department of Electrical Engineering, Chang Gung University, 259 WenHua 1st Road, Taoyuan County, Taoyuan, Taiwan \\ ${ }^{6}$ Department of Biomedical Engineering, University at Buffalo, The State University of New York, Buffalo, NY 14260, USA \\ ${ }^{7}$ Department of Electrical Engineering, University at Buffalo, The State University of New York, Buffalo, NY 14260, USA \\ ${ }^{8}$ Neuroscience Research Center, Chang Gung Memorial Hospital, 5 FuHsing Street, Linkou, Taoyuan County, Taiwan \\ ${ }^{9}$ Institute of Radiological Research, Chang Gung University, Chang Gung Memorial Hospital, 5 FuHsing Street, \\ Linkou, Taoyuan County, Taiwan \\ ${ }^{10}$ Healthy Aging Research Center, Chang Gung University, 259 WenHua 1st Road, Taoyuan County, Taoyuan, Taiwan
}

Correspondence should be addressed to Jiun-Jie Wang; jwang@mail.cgu.edu.tw

Received 10 January 2014; Accepted 10 April 2014; Published 12 May 2014

Academic Editor: Hengyi Rao

Copyright (C) 2014 Yau-Yau Wai et al. This is an open access article distributed under the Creative Commons Attribution License, which permits unrestricted use, distribution, and reproduction in any medium, provided the original work is properly cited.

\begin{abstract}
Rationale and Objectives. The primary objective of the current investigation was to characterize white matter integrity in different subtypes of mild cognitive impairment (MCI) using tract-based spatial statistics of diffusion tensor imaging. Materials and Methods. The study participants were divided into 4 groups of 30 subjects each as follows: cognitively healthy controls, amnestic MCI, dysexecutive MCI, and Alzheimer's disease (AD). All subjects underwent a comprehensive neuropsychological assessment, apolipoprotein E genotyping, and 3-tesla MRI. The diffusion tensor was reconstructed and then analyzed using tract-based spatial statistics. The changes in brain white matter tracts were also examined according to the apolipoprotein E $\varepsilon 4$ status. Results. Compared with controls, amnestic MCI patients showed significant differences in the cerebral white matter, where changes were consistently detectable in the frontal and parietal lobes. We found a moderate impact of the apolipoprotein $\mathrm{E} \varepsilon 4$ status on the extent of white matter disruption in the amnestic MCI group. Patients with AD exhibited similar but more extensive alterations, while no significant changes were observed in dysexecutive MCI patients. Conclusion. The results from this study indicate that amnestic $\mathrm{MCI}$ is the most likely precursor to $\mathrm{AD}$ as both conditions share significant white matter damage. By contrast, dysexecutive $\mathrm{MCI}$ seems to be characterized by a distinct pathogenesis.
\end{abstract}

\section{Introduction}

Dementia refers to a clinical syndrome of acquired intellectual disturbances produced by brain dysfunction [1]. Mild cognitive impairment (MCI) is considered to be the clinical transition stage between normal aging and dementia [2].
Evidence suggests that subjects with MCI tend to progress to probable Alzheimer's disease (AD) at a rate of approximately $10 \%$ to $15 \%$ per year [2]. However, MCI is a clinically heterogeneous syndrome, with some patients showing isolated memory impairments (i.e., amnestic MCI) and others with isolated executive function impairments (i.e., dysexecutive 
MCI). Importantly, Yaffe et al. [3] demonstrated that dysexecutive MCI patients are less likely to convert to dementia but have higher 5-year mortality rates than amnestic MCI.

MRI studies of dementia have been mainly focused on the assessment of hippocampal and entorhinal atrophy [46]. Global brain atrophy on conventional MRI has been proposed as a marker for advanced MCI [7, 8], but the low specificity limits its clinical value $[9,10]$. Similarly, neuropsychological tests can be considered as screening tools for MCI but are not adequate for diagnosis. Diffusion tensor imaging (DTI) is MRI technique that can noninvasively measure macroscopic axonal organization in the central nervous system $[11,12]$. Because of the potential for investigating white matter integrity and fiber connectivity in vivo, this technique has been widely applied to study brain disorders $[13,14]$.

Three eigenvalues and three corresponding eigenvectors can be derived from the diffusion tensor. The largest eigenvalue is often referred to as the axial diffusivity (longitudinal diffusivity (LD), in the study, in order not to be confused with Alzheimer's disease) and the average of the second and third eigenvalues as radial diffusivity (RD) [15]. In white matter, diffusion tensor is usually modeled as cylindrically symmetric $[16,17]$. The increase of the mean diffusivity (MD) can be related to an increase in either LD or RD. The reduction of FA can be related to either a decrease of diffusion in the longitudinal direction (AD) or an increase in the transverse direction (RD). Many studies attributed the changes in $\mathrm{RD}$ as related axonal injury or demyelination process [18].

Conventional DTI data are often analyzed in a region of interest approach. Because the directional information is encoded by the signal intensity, great care is required for all imaging processing procedures (e.g., registration and normalization). Tract-based spatial statistics (TBSS) $[19,20]$ is a new whole brain voxelwise analysis method that provides more reliable alignment of the white matter tracts and less bias from smoothing than conventional voxel-based methods. The data-driven approach of TBSS is particularly useful in a disorder like MCI, for which regional patterns of brain abnormalities are not fully determined. Thus, the aim of the present study was to investigate white matter changes in two MCI subgroups, dysexecutive MCI and amnestic MCI, using a TBSS approach. Imaging patterns were also compared with those of $\mathrm{AD}$ patients and cognitively healthy controls.

\section{Materials and Methods}

The study was approved by our institutional review board and complied with the tenets of the Declaration of Helsinki. Each participant or participant's legal guardian gave written informed consent for participation.

2.1. Participants and Clinical Workup. The study participants $(n=120)$ were divided into 4 clinical groups of 30 subjects each as follows: cognitively healthy controls, amnestic MCI, dysexecutive MCI, and AD. The clinical workup included a thorough medical history, physical examination, and neuropsychological testing. Four key cognitive domains were assessed: memory, executive function, language, and visuospatial skills. All subjects were screened for the presence of depressive symptoms using either the Hamilton depression rating scale (healthy control) or the Cornell scale for depression in dementia (AD, amnestic MCI, and dysexecutive MCI). Patients with depression were excluded.

The diagnosis of AD was made using the NINCDSADRDA criteria [21]. The clinical dementia rating was used to quantify the severity of symptoms of dementia. MCI patients were diagnosed after an extensive clinical evaluation. The clinical phenotype of MCI was determined according to the criteria by Petersen [1]. The amnestic MCI group exhibited an isolated memory impairment without deficits in other cognitive domains. Patients with dysexecutive MCI had a relatively focal dysfunction in the executive domain, while memory, language, and visuospatial skills remained within the normal range. Cognitively healthy controls were free of cognitive impairment as judged by clinical assessment, neuropsychological testing, and clinical dementia rating.

2.2. Apolipoprotein E Genotyping. Genomic DNA was extracted from leukocytes in samples of whole blood, following a standard salting-out technique. Apolipoprotein E genotypes were detected by polymerase chain reaction followed by restriction fragment length polymorphism analysis.

2.3. Image Acquisition. Images were acquired using a 3-tesla MR scanner (Magnetom Trio with TIM, Siemens, Erlangen, Germany). T2WI FLAIR and T1WI magnetization-prepared rapid acquisition gradient echo images were acquired to rule out concomitant neurological disorders. DTI was acquired using a spin-echo EPI sequence with the following parameters: TR/TE/flip angle $=7500 \mathrm{~ms} / 83 \mathrm{~ms} / 90^{\circ}$, field of view $=$ $256 \mathrm{~mm}^{2}$, and matrix size $=128 \times 128$. Diffusion-weighting gradients were applied in 64 noncollinear directions with a diffusion weighting of $1000 \mathrm{~s} / \mathrm{mm}^{2}$. Sixty-four contiguous axial slices were obtained to cover the majority of the brain with an isotropic voxel size of $2 \mathrm{~mm}$. The single average acquisition time was 8 minutes 47 seconds.

2.4. Image Processing and Analysis. Diffusion analysis was performed and the white matter skeleton was identified using TBSS, part of FSL (http://fsl.fmrib.ox.ac.uk/fsl/ fsl4.0/tbss/index). In brief, the reconstructed fractional anisotropy $[16,17]$ of each individual was initially aligned to the MNI template. The parameters from the nonlinear registration were then applied to the diffusion indices, including $\mathrm{MD} / \mathrm{LD} / \mathrm{RD}[16,17]$. The spatial normalization was achieved by 12-parameter affine transformation. Both followed the recommended procedures and parameters by TBSS. The aligned diffusion indices were then projected onto a fiber tract-derived skeleton and fed into the voxelwise crosssubject statistics. Data from each of the diffusion indices were compared by the Randomise 2.1 software [22, 23]. Multiple comparison correction was done by the familywise error correction with a threshold of $P<0.05$. We identified abnormal white matter tracts based on the atlas prepared 
TABLE 1: General characteristics of the study participants.

\begin{tabular}{|c|c|c|c|c|}
\hline & Controls & Dysexecutive & Amnestic & $\mathrm{AD}$ \\
\hline Number of subjects & 30 & 30 & 30 & 30 \\
\hline Age (years) & $67.2 \pm 5.6$ & $65.9 \pm 7.6$ & $68.6 \pm 8.3$ & $73.9 \pm 9.4$ \\
\hline Range & $61-80$ & $50-84$ & $56-84$ & $54-83$ \\
\hline Median & 65.5 & 65 & 69 & 76.5 \\
\hline Sex (males/females) & $18 / 12$ & $12 / 18$ & $17 / 13$ & $12 / 18$ \\
\hline Education (years) & $10.1 \pm 4.7$ & $8.0 \pm 3.5$ & $8.3 \pm 5.3$ & $7.3 \pm 6.4$ \\
\hline Clinical dementia rating score & $0.00 \pm 0.00$ & $0.12 \pm 0.22$ & $0.47 \pm 0.13$ & $1.00 \pm 0.57$ \\
\hline \multicolumn{5}{|l|}{ Apolipoprotein E status } \\
\hline$\varepsilon 4$ carriers & 2 & 4 & 11 & 5 \\
\hline$\varepsilon 4$ noncarriers & 28 & 23 & 16 & 23 \\
\hline Unknown & 0 & 3 & 3 & 2 \\
\hline
\end{tabular}

Data are given as means \pm standard deviation or counts, as appropriate.

TABLE 2: Neuropsychological test scores.

\begin{tabular}{|c|c|c|c|c|c|c|}
\hline & Controls & $\begin{array}{c}\text { Apolipoprotein } \\
\text { E } \varepsilon 4 \\
\text { noncarriers } \\
\end{array}$ & $\begin{array}{c}\text { Amnestic MCI } \\
\text { apolipoprotein E } \varepsilon 4 \text { carriers }\end{array}$ & Sum & $\begin{array}{l}\text { Dysexecutive } \\
\text { MCI }\end{array}$ & $\mathrm{AD}$ \\
\hline \multicolumn{7}{|l|}{ Mean global score } \\
\hline $\begin{array}{l}\text { Mini-mental status } \\
\text { examination }\end{array}$ & $28.23(1.30)$ & $25.25(3.87)$ & $25.09(4.18)$ & $25.10(3.87)$ & $27.63(1.47)$ & $15.07(6.34)$ \\
\hline \multicolumn{7}{|l|}{ Mean memory score } \\
\hline Word sequence learning-recall & $3.17(1.93)$ & $0.47(0.64)$ & $0.55(0.93)$ & $0.45(0.74)$ & $2.00(1.68)$ & $0.04(0.20)$ \\
\hline Logic memory II & $11.60(2.72)$ & $8.25(3.66)$ & $8.18(4.21)$ & $7.97(3.72)$ & $9.87(2.42)$ & $3.15(1.87)$ \\
\hline \multicolumn{7}{|l|}{ Mean executive function score } \\
\hline $\begin{array}{l}\text { Semantic association of verbal } \\
\text { fluency }\end{array}$ & $33.40(6.82)$ & $31.81(4.04)^{*}$ & $25.73(7.16)^{*}$ & $29.57(6.03)$ & $32.83(7.37)$ & $14.55(6.20)$ \\
\hline $\begin{array}{l}\text { Wisconsin card sorting test } \\
\text { Completed Categories }\end{array}$ & $5.20(1.13)$ & $3.67(1.91)$ & $3.82(1.89)$ & $3.69(1.77)$ & $2.60(1.35)$ & $1.88(2.76)$ \\
\hline \multicolumn{7}{|l|}{ Mean visuospatial score } \\
\hline 3D block construction models & $28.6(0.77)$ & $27.31(2.60)$ & $26.91(4.11)$ & $27.27(3.1)$ & $28.03(1.65)$ & $18.41(10.50)$ \\
\hline \multicolumn{7}{|l|}{ Mean language score } \\
\hline Object naming test & $16.00(0.00)$ & $15.94(0.25)$ & $16.00(0.00)$ & $15.97(0.18)$ & $15.97(0.18)$ & $13.13(3.65)$ \\
\hline
\end{tabular}

Data are given as means (standard deviation); ${ }^{*}$ Significant differences at $P<0.05(P=0.0225)$.

at Johns Hopkins University [24]. The correlation between neuropsychiatry measures and the diffusion indices was assessed using the regression model in the SPM (Wellcome Trust Centre for Neuroimaging, UK). The effect of the apolipoprotein $\mathrm{E} \varepsilon 4$ allele was determined by dividing participants in either apolipoprotein E $\varepsilon 4(\varepsilon 2 / \varepsilon 4, \varepsilon 3 / \varepsilon 4$, and $\varepsilon 4 / \varepsilon 4)$ or non- $\varepsilon 4(\varepsilon 2 / \varepsilon 2, \varepsilon 2 / \varepsilon 3$, and $\varepsilon 3 / \varepsilon 3)$ carriers. Cognitive performances were compared between carriers and noncarriers using Student's $t$-test. The TBSS approach was also applied to compare the imaging patterns according to the apolipoprotein $\mathrm{E} \varepsilon 4$ carrier status.

\section{Results}

Table 1 shows the general characteristics of the study participants. The neuropsychological tests are reported in Table 2. The results from TBSS analysis did not identify significant white matter changes in dysexecutive MCI patients compared with cognitively healthy controls. Figure 1 shows the changes in fractional anisotropy along the white matter skeleton of patients with amnestic MCI (a) and AD (b) compared with healthy controls. Both groups of patients exhibited a widespread fractional anisotropy decrease within the white matter tracts. Changes in the amnestic MCI group were restricted to the frontal and parietal lobes, while $\mathrm{AD}$ patients consistently exhibited more extensive damage which was suggestive of a progressive disease process.

Figure 2 shows an increase in RD along the white matter skeleton of both amnestic MCI (a) and AD (b) patients compared with healthy controls. The location was consistent but significantly smaller than the corresponding areas identified in Figure 1. The arrows indicate the location of the most significant areas of increased RD. These results suggest that an increased $\mathrm{RD}$ was presumably driving a reduction of fractional anisotropy in both $\mathrm{AD}$ and amnestic MCI patients. 


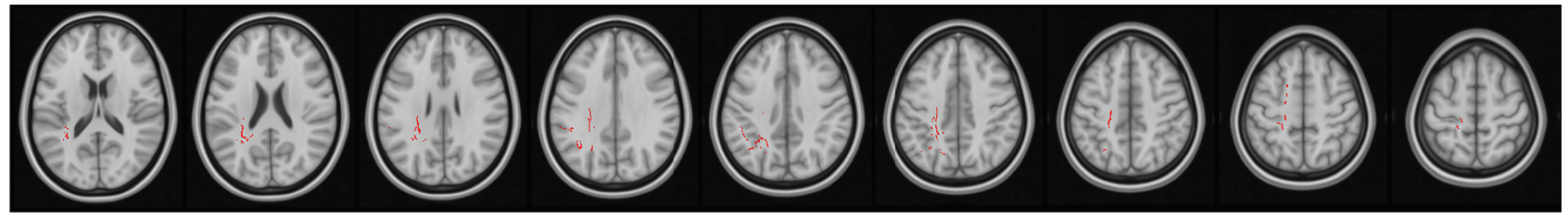

(a)

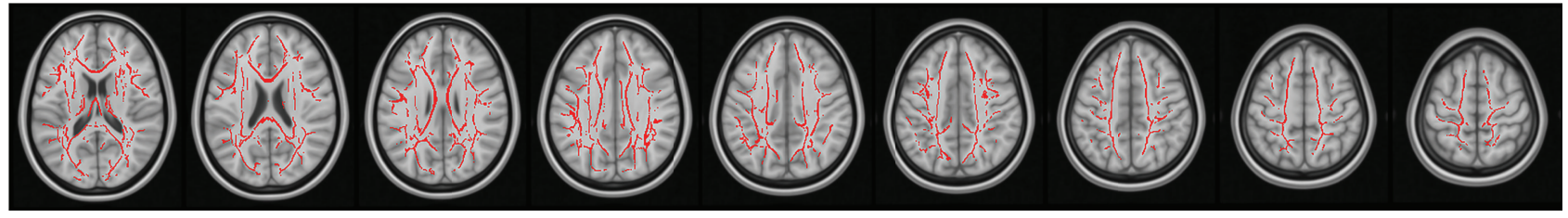

(b)

FIGURE 1: Fractional anisotropy in the white matter regions of the study participants. Compared with normal controls, fractional anisotropy is reduced within the white matter skeleton of both patients with amnestic MCI (a) and AD (b). The left of the figure corresponds to the subject's right side.

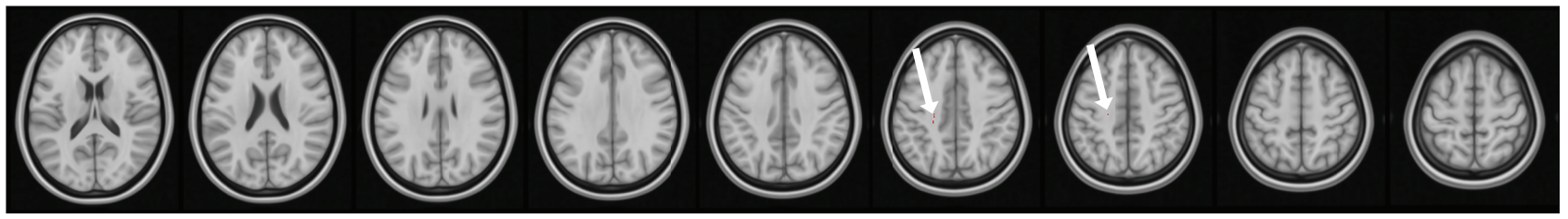

(a)

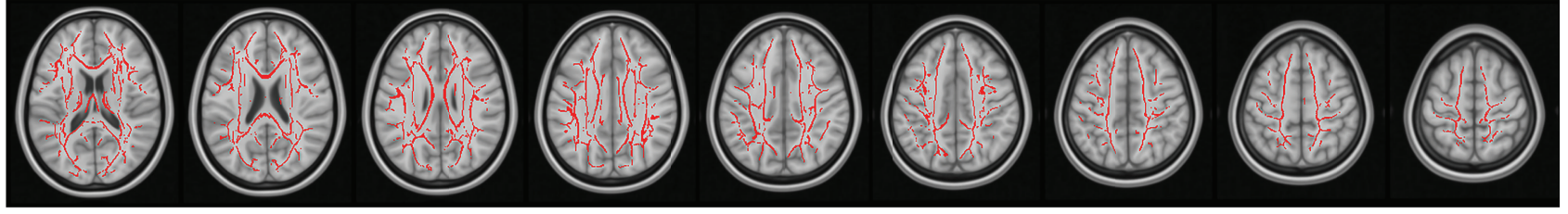

(b)

FIGURE 2: Radial diffusivity in the white matter regions of the study participants. Compared with normal controls, radial diffusivity is increased within the white matter skeleton of both patients with amnestic MCI (a) and AD (b). The pattern of such changes is consistent with the observed reductions in fractional anisotropy. The arrows indicate the locations of the fiber tracts showing significant changes.

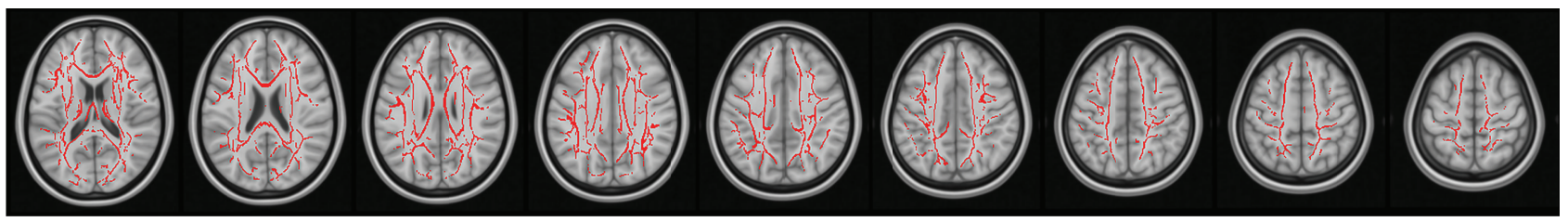

(a)

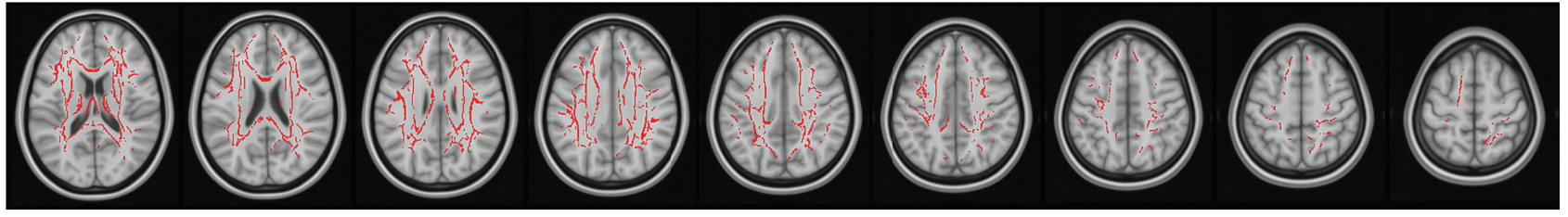

(b)

FIGURE 3: MD and longitudinal diffusivity in the white matter regions in patients with AD. Compared with normal controls, MD (a) and longitudinal diffusivity (b) are significantly increased within the white matter skeleton of patients with AD. The observed increases are largely consistent with the corresponding reductions in fractional anisotropy. 


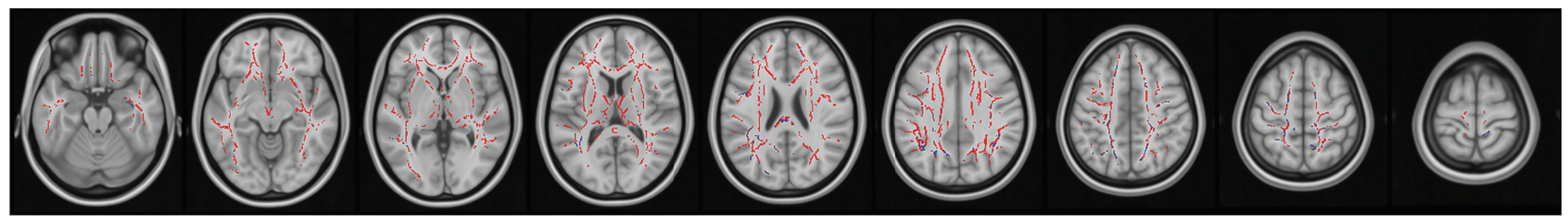

(a)

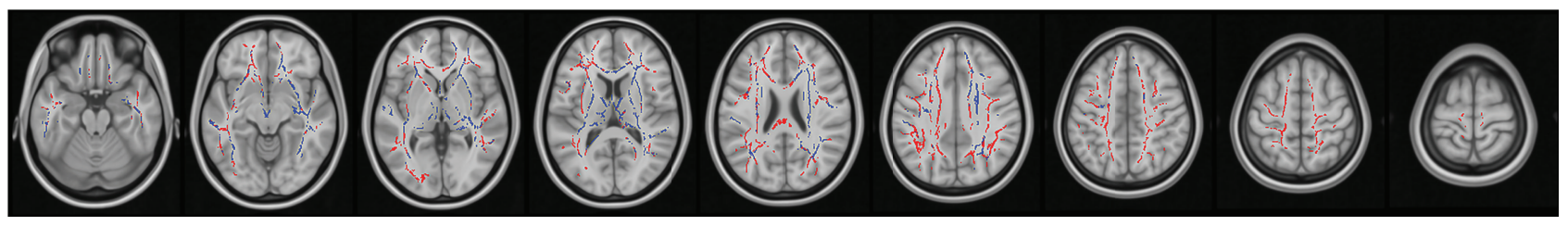

(b)

FIGURE 4: Effect of the apolipoprotein $\mathrm{E} \varepsilon 4$ status on white matter changes in amnestic MCI patients. The diffusion indices are examined in amnestic MCI patients after stratification according to apolipoprotein E $\varepsilon 4$ status. In (a), the decrease of fractional anisotropy is marked in blue, while the increase in MD is reported in red. In (b), the increases of $\mathrm{LD}$ and $\mathrm{RD}$ are marked in blue and red, respectively.

Figure 3 shows the corresponding increases of MD (a) and $\mathrm{LD}(\mathrm{b})$ in $\mathrm{AD}$ patients. No significant changes of these parameters were found in the amnestic MCI group. The observed increase of $\mathrm{MD}$ in $\mathrm{AD}$ patients was largely in accordance with the corresponding fractional anisotropy changes (depicted in Figure 1(b)). The pattern of LD increases was similarly consistent, with the only exception being the absence of significant changes in the occipital lobe.

The effect of the apolipoprotein E $\varepsilon 4$ allele on white matter changes in amnestic MCI patients is shown in Figure 4. In (a), the decrease of fractional anisotropy is marked in blue, while the increase in MD is reported in red. In (b), the increases of $\mathrm{LD}$ and $\mathrm{RD}$ are marked in blue and red, respectively. Compared with healthy subjects, amnestic MCI patients without the apolipoprotein E $\varepsilon 4$ allele exhibited larger and more striking changes in both fractional anisotropy and RD. Similarly, more extensive areas of MD and LD changes were evident in apolipoprotein $\mathrm{E} \varepsilon 4$ noncarriers.

In all study groups, we found no correlation between changes in diffusion indices and neuropsychiatric measurements, even after allowance for age and/or education levels.

\section{Discussion}

There are three main findings in this study. First, we have shown that $\mathrm{AD}$ and amnestic MCI may share a common neurodegenerative substrate which impairs white matter integrity. The results from this study indicate that this process can be efficiently visualized in a noninvasive manner using DTI-TBSS. Second, AD patients showed more extensive white matter damage compared with amnestic MCI subjects, supporting the view that amnestic MCI is the most likely precursor to $\mathrm{AD}$. Third, the apolipoprotein $\mathrm{E} \varepsilon 4$ carrier status affected the extent of white matter involvement in amnestic MCI patients. Importantly, we found no evidence of significant white matter damage in dysexecutive MCI, suggesting highly distinct pathways of neurodegeneration compared with amnestic MCI. Moreover, there was no correlation between diffusion indices and neuropsychological test scores, suggesting no direct association between DTI-TBSS findings and MCI.

4.1. White Matter Changes in AD and MCI. Diffusion tensor imaging is sensitive to white matter damage and has been previously used to examine entire neural networks and their integrity in $\mathrm{AD}$ and MCI $[25,26]$. The observed changes in diffusion indices have been mainly attributed to alterations in the white matter structure. Compared with normal controls, both amnestic MCI and $\mathrm{AD}$ are characterized by increases in $\mathrm{RD}$ and $\mathrm{MD}$ and a reduction in fractional anisotropy. The increase in RD has generally been ascribed to alterations in myelin sheaths, which can be related to the observed changes in both MD and fractional anisotropy. As these indices are altered in amnestic MCI and AD, it is feasible that both conditions are characterized by an altered brain myelination.

The results from this study indicate a significant overlap between the affected regions of amnestic MCI and AD patients. The changes in amnestic MCI patients were chiefly located in the frontal and parietal regions. However, the extension of damage was significantly greater in $\mathrm{AD}$ patients, suggesting that amnestic MCI is the most likely precursor to $\mathrm{AD}$.

LD did not differ in both amnestic and dysexecutive MCI, while $\mathrm{AD}$ patients showed an increase of both $\mathrm{LD}$ and $\mathrm{RD}$. In normal aging, an increased LD reflects a reduced axonal packing in white matter structures due to thinning of myelin or decrease in axonal diameter. This phenomenon in turn results in an increased diffusivity in all orientations within a voxel $[25,27]$. Using MRS, Meyrhoff et al. provided suggestive evidence of diffuse axonal injury and membrane alterations in $\mathrm{AD}$ patients [28]. Our results indicate that LD changes seem to be restricted to $\mathrm{AD}$ patients.

We found no significant association between neuropsychological testing and the results of DTI-TBSS analysis. 
However, these results should be interpreted cautiously as cognitive performances were assessed only at the study admission; additionally, all neuropsychological scores were distributed in a narrow range. In any case, it is noteworthy that white matter changes on DTI-TBSS do not necessarily reflect changes in cortical functions.

In this study, there were no significant alterations in diffusion indices of dysexecutive MCI patients, suggesting that changes in white matter structure do not play a major role in this condition. Alternatively, such alterations could be so subtle that they escaped detection by means of DTITBSS. Further studies are necessary to shed more light on the neuroradiological alterations specific to dysexecutive MCI.

4.2. Impact of APOE \&4 Carrier Status on White Matter Changes. The apolipoprotein E $\varepsilon 4$ allele is the most widely recognized genetic risk factor for sporadic AD [29] and affects, even for nondemented elders, the levels of cognitive performance [30]. In the present study, amnestic MCI patients who carried at least one apolipoprotein E $\varepsilon 4$ allele showed a nonsignificant trend toward lower scores on the executive function and memory measures compared with those without. In contrast, we found no impact of the apolipoprotein E $\varepsilon 4$ status on both visuospatial and language processing.

The results from the DTI-TBSS analysis demonstrated that the apolipoprotein E $\varepsilon 4$ status had a moderate impact on the extent of white matter disruption in amnestic MCI patients. In subjects without the apolipoprotein E $\varepsilon 4$ allele, we found that $\mathrm{RD}$ and $\mathrm{LD}$ were significantly increased, which can be related to the observation of an increased MD and a reduced fractional anisotropy. It is noteworthy that the impact of the apolipoprotein E $\varepsilon 4$ status on cognitive functions may vary in different ethnic groups [31, 32]. Interestingly, the apolipoprotein E $\varepsilon 4$ allele is less prevalent in people of Chinese ancestry [33]. This variability has been shown to influence the association of this polymorphism with different phenotypes $[33,34]$. For this reason, the observed association between the apolipoprotein $\mathrm{E} \varepsilon 4$ status and the extent of white matter disruption in our amnestic MCI patients should be confirmed in multiethnic populations. In addition, future studies should assess how the presence of white matter abnormalities reflects associative cortical functioning in MCI patients.

4.3. Comparison with Previous Studies. Bosch et al. [35] have previously identified a general neural network which seems to be specifically disrupted in $\mathrm{AD}$. However, the authors failed to identify specific white matter changes in amnestic MCI patients compared with healthy controls. In contrast, Zhuang et al. [36] reported a significant fractional anisotropy reduction in the frontal, temporal, and parietal lobes of amnestic MCI patients. The fractional anisotropy changes identified in our study are similar to those reported by Zhuang et al. [36], albeit being located in a smaller region. This discrepancy can be attributed to the different sample sizes used in the studies. It is also important to note that MCI is not a diagnostic entity as $\mathrm{AD}$ is; consequently, a number of studies use the term MCI but do not use Petersen's criteria [1]. Between-study heterogeneity in MCI severity may also explain the discrepancy. In accordance with Zhuang et al. [36], our study identified significant signal changes of the white matter in amnestic MCI patients. This result deserves further independent scrutiny.

4.4. Study Limitations. The findings of our study should be interpreted within the context of the following limitations. First, a well-established model for tensor signal processing in the gray matter is still lacking. For this reason, we limited our DTI-TBSS analysis to the white matter skeleton. The analysis of gray matter diffusion properties may be challenging, but this knowledge may ultimately help define the correlation between white matter changes and cortical functioning in amnestic MCI and AD patients. Furthermore, the observed changes in $\mathrm{LD} / \mathrm{RD}$, as detected in the white matter skeleton, can be controversial noticeably in the region where the fiber distribution is complicated or where the fibers cross [37, 38]. Therefore, the interpretation of the observed white matter change should be exercised with great care.

Second, in this study we did not correlate in vivo imaging findings with postmortem analysis of brain tissue. Furthermore, given the cross-sectional design, we cannot infer causality between white matter changes and the conversion of amnestic MCI to AD. Notwithstanding these limitations, the results from this study indicate that amnestic MCI is the most likely precursor to $\mathrm{AD}$ as both conditions share significant white matter damage. By contrast, dysexecutive MCI seems to be characterized by a distinct pathogenetic mechanism.

\section{Conclusion}

Amnestic MCI is the most likely precursor to Alzheimer's disease because both conditions share significant white matter damage as assessed by tract-based spatial statistics. Dysexecutive MCI seems to be characterized by a distinct pathogenesis.

\section{Conflict of Interests}

The authors declare that there is no conflict of interests regarding the publication of this paper.

\section{Authors' Contribution}

Yau-Yau Wai and Wen-Chiun Hsu contributed equally to the paper.

\section{Acknowledgments}

This work was supported by the Ministry of Science and Technology, Taiwan (102-2325-B-182-008) and Chang Gung Memorial Hospital, Linkou (CMRPD1C0292 and CMRPD3D0011). The imaging facility was supported by the Molecular Imaging Center and Neuroscience Research Center, Chang Gung Memorial Hospital, Linkou. The funding source had no involvement in the collection, analysis, and 
interpretation of data; in the writing of the report; or in the decision to submit the paper for publication.

\section{References}

[1] R. C. Petersen, "Mild cognitive impairment as a diagnostic entity," Journal of Internal Medicine, vol. 256, no. 3, pp. 183-194, 2004.

[2] J. Pa, A. Boxer, L. L. Chao et al., "Clinical-neuroimaging characteristics of dysexecutive mild cognitive impairment," Annals of Neurology, vol. 65, no. 4, pp. 414-423, 2009.

[3] K. Yaffe, R. C. Petersen, K. Lindquist, J. Kramer, and B. Miller, "Subtype of mild cognitive impairment and progression to dementia and death," Dementia and Geriatric Cognitive Disorders, vol. 22, no. 4, pp. 312-319, 2006.

[4] A. Convit, J. De Asis, M. J. De Leon, C. Y. Tarshish, S. De Santi, and H. Rusinek, "Atrophy of the medial occipitotemporal, inferior, and middle temporal gyri in non-demented elderly predict decline to Alzheimer's disease," Neurobiology of Aging, vol. 21, no. 1, pp. 19-26, 2000.

[5] A. Convit, M. J. De Leon, C. Tarshish et al., "Specific hippocampal volume reductions in individuals at risk for Alzheimer's disease," Neurobiology of Aging, vol. 18, no. 2, pp. 131-138, 1997.

[6] J. L. Whitwell, M. M. Shiung, S. A. Przybelski et al., "MRI patterns of atrophy associated with progression to $\mathrm{AD}$ in amnestic mild cognitive impairment," Neurology, vol. 70, no. 7, pp. 512-520, 2008.

[7] S. M. de la Monte, "Quantitation of cerebral atrophy in preclinical and end-stage Alzheimer's disease," Annals of Neurology, vol. 25, no. 5, pp. 450-459, 1989.

[8] M. J. de Leon, J. Golomb, A. E. George et al., “The radiologic prediction of Alzheimer disease: the atrophic hippocampal formation," American Journal of Neuroradiology, vol. 14, no. 4, pp. 897-906, 1993.

[9] G. D. Jackson, R. I. Kuzniecky, and G. D. Cascino, "Hippocampal sclerosis without detectable hippocampal atrophy," Neurology, vol. 44, no. 1, pp. 42-46, 1994.

[10] A. Pitkänen, M. Laakso, R. Kälviäinen et al., "Severity of hippocampal atrophy correlates with the prolongation of MRI $\mathrm{T}_{2}$ relaxation time in temporal lobe epilepsy but not in Alzheimer's disease," Neurology, vol. 46, no. 6, pp. 1724-1730, 1996.

[11] S. G. Horovitz, P. Skudlarski, and J. C. Gore, "Correlations and dissociations between BOLD signal and P300 amplitude in an auditory oddball task: a parametric approach to combining fMRI and ERP," Magnetic Resonance Imaging, vol. 20, no. 4, pp. 319-325, 2002.

[12] B. W. Müller, P. Stude, K. Nebel et al., "Sparse imaging of the auditory oddball task with functional MRI," NeuroReport, vol. 14, no. 12, pp. 1597-1601, 2003.

[13] M. Fushimi, N. Matsubuchi, and A. Sekine, "Progression of P300 in a patient with bilateral hippocampal lesions," Clinical Neurophysiology, vol. 116, no. 3, pp. 625-631, 2005.

[14] E. B. Myers and S. E. Blumstein, "The neural bases of the lexical effect: an fMRI investigation," Cerebral Cortex, vol. 18, no. 2, pp. 278-288, 2008.

[15] S.-K. Song, S.-W. Sun, M. J. Ramsbottom, C. Chang, J. Russell, and A. H. Cross, "Dysmyelination revealed through MRI as increased radial (but unchanged axial) diffusion of water," NeuroImage, vol. 17, no. 3, pp. 1429-1436, 2002.

[16] P. J. Basser and C. Pierpaoli, "Microstructural and physiological features of tissues elucidated by quantitative-diffusion-tensor
MRI," Journal of Magnetic Resonance B, vol. 111, no. 3, pp. 209219, 1996.

[17] C. Pierpaoli and P. J. Basser, "Toward a quantitative assessment of diffusion anisotropy," Magnetic Resonance in Medicine, vol. 36, no. 6, pp. 893-906, 1996.

[18] M. D. Budde, M. Xie, A. H. Cross, and S.-K. Song, "Axial diffusivity is the primary correlate of axonal injury in the experimental autoimmune encephalomyelitis spinal cord: a quantitative pixelwise analysis," The Journal of Neuroscience, vol. 29, no. 9, pp. 2805-2813, 2009.

[19] S. M. Smith, H. Johansen-Berg, M. Jenkinson et al., "Acquisition and voxelwise analysis of multi-subject diffusion data with tract-based spatial statistics," Nature Protocols, vol. 2, no. 3, pp. 499-503, 2007.

[20] S. M. Smith, M. Jenkinson, H. Johansen-Berg et al., "Tractbased spatial statistics: voxelwise analysis of multi-subject diffusion data," NeuroImage, vol. 31, no. 4, pp. 1487-1505, 2006.

[21] G. McKhann, D. Drachman, M. Folstein, R. Katzman, D. Price, and E. M. Stadlan, "Clinical diagnosis of Alzheimer's disease: report of the NINCDS-ADRDA work group under the auspices of Department of Health and Human Services Task Force on Alzheimer's disease," Neurology, vol. 34, no. 7, pp. 939-944, 1984.

[22] E. T. Bullmore, J. Suckling, S. Overmeyer, S. Rabe-Hesketh, E. Taylor, and M. J. Brammer, "Global, voxel, and cluster tests, by theory and permutation, for a difference between two groups of structural mr images of the brain," IEEE Transactions on Medical Imaging, vol. 18, no. 1, pp. 32-42, 1999.

[23] T. E. Nichols and A. P. Holmes, "Nonparametric permutation tests for functional neuroimaging: a primer with examples," Human Brain Mapping, vol. 15, no. 1, pp. 1-25, 2002.

[24] S. Wakana, H. Jiang, L. M. Nagae-Poetscher, P. C. M. van Zijl, and S. Mori, "Fiber tract-based atlas of human white matter anatomy," Radiology, vol. 230, no. 1, pp. 77-87, 2004.

[25] E. V. Sullivan, T. Rohlfing, and A. Pfefferbaum, "Quantitative fiber tracking of lateral and interhemispheric white matter systems in normal aging: relations to timed performance," Neurobiology of Aging, vol. 31, no. 3, pp. 464-481, 2010.

[26] A. Stadlbauer, E. Salomonowitz, G. Strunk, T. Hammen, and O. Ganslandt, "Age-related degradation in the central nervous system: assessment with diffusion-tensor imaging and quantitative fiber tracking," Radiology, vol. 247, no. 1, pp. 179-188, 2008.

[27] C. D. Smith, H. Chebrolu, D. R. Wekstein, F. A. Schmitt, and W. R. Markesbery, "Age and gender effects on human brain anatomy: a voxel-based morphometric study in healthy elderly," Neurobiology of Aging, vol. 28, no. 7, pp. 1075-1087, 2007.

[28] D. J. Meyerhoff, S. MacKay, J.-M. Constans et al., "Axonal injury and membrane alterations in Alzheimer's disease suggested by in vivo proton magnetic resonance spectroscopic imaging," Annals of Neurology, vol. 36, no. 1, pp. 40-47, 1994.

[29] X. He, K. Cooley, C. H. Y. Chung, N. Dashti, and J. Tang, "Apolipoprotein receptor 2 and X11 $\alpha / \beta$ mediate apolipoprotein E-induced endocytosis of amyloid- $\beta$ precursor protein and $\beta$ secretase, leading to amyloid- $\beta$ production," The Journal of Neuroscience, vol. 27, no. 15, pp. 4052-4060, 2007.

[30] R. A. Honea, E. Vidoni, A. Harsha, and J. M. Burns, "Impact of APOE on the healthy aging brain: a voxel-based MRI and DTI study," Journal of Alzheimer's Disease, vol. 18, no. 3, pp. 553-564, 2009.

[31] G. I. Korovaitseva, T. V. Sherbatich, N. V. Selezneva et al., "Association between the apolipoprotein E (APOE) gene and various forms of Alzheimer's disease," Genetika, vol. 37, no. 4, pp. 529-535, 2001. 
[32] S. G. Post, P. J. Whitehouse, R. H. Binstock et al., "The clinical introduction of genetic testing for Alzheimer disease: an ethical perspective," The Journal of the American Medical Association, vol. 277, no. 10, pp. 832-836, 1997.

[33] S. Y. S. Wong, E. M. C. Lau, M. Li, T. Chung, A. Sham, and J. Woo, "The prevalence of Apo E4 genotype and its relationship to bone mineral density in Hong Kong Chinese," Journal of Bone and Mineral Metabolism, vol. 23, no. 3, pp. 261-265, 2005.

[34] H.-C. Liu, C. J. Hong, S. J. Wang et al., "ApoE genotype in relation to AD and cholesterol: a study of 2,326 Chinese adults," Neurology, vol. 53, no. 5, pp. 962-966, 1999.

[35] B. Bosch, E. M. Arenaza-Urquijo, L. Rami et al., "Multiple DTI index analysis in normal aging, amnestic MCI and AD. Relationship with neuropsychological performance," Neurobiology of Aging, vol. 33, no. 1, pp. 61-74, 2012.

[36] L. Zhuang, W. Wen, W. Zhu et al., "White matter integrity in mild cognitive impairment: a tract-based spatial statistics study," NeuroImage, vol. 53, no. 1, pp. 16-25, 2010.

[37] D. K. Jones, T. R. Knosche, and R. Turner, "White matter integrity, fiber count, and other fallacies: the do's and don'ts of diffusion MRI," Neuroimage, vol. 73, pp. 239-254, 2013.

[38] C. A. M. Wheeler-Kingshott and M. Cercignani, "About "axial" and "radial" diffusivities," Magnetic Resonance in Medicine, vol. 61, no. 5, pp. 1255-1260, 2009. 


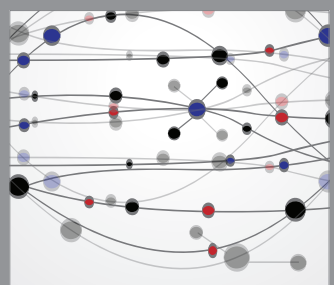

The Scientific World Journal
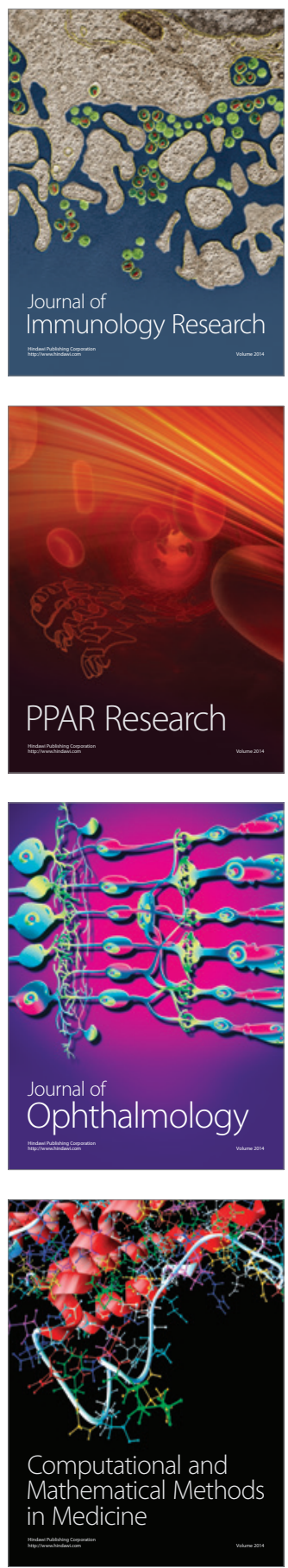

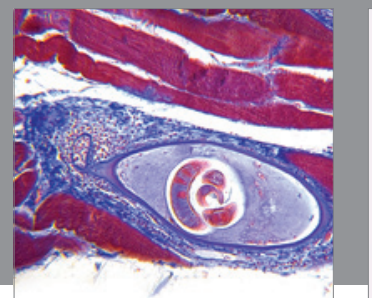

Gastroenterology

Research and Practice
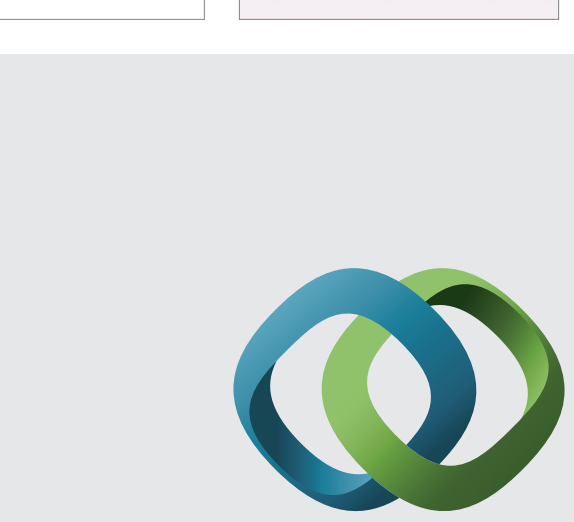

\section{Hindawi}

Submit your manuscripts at

http://www.hindawi.com
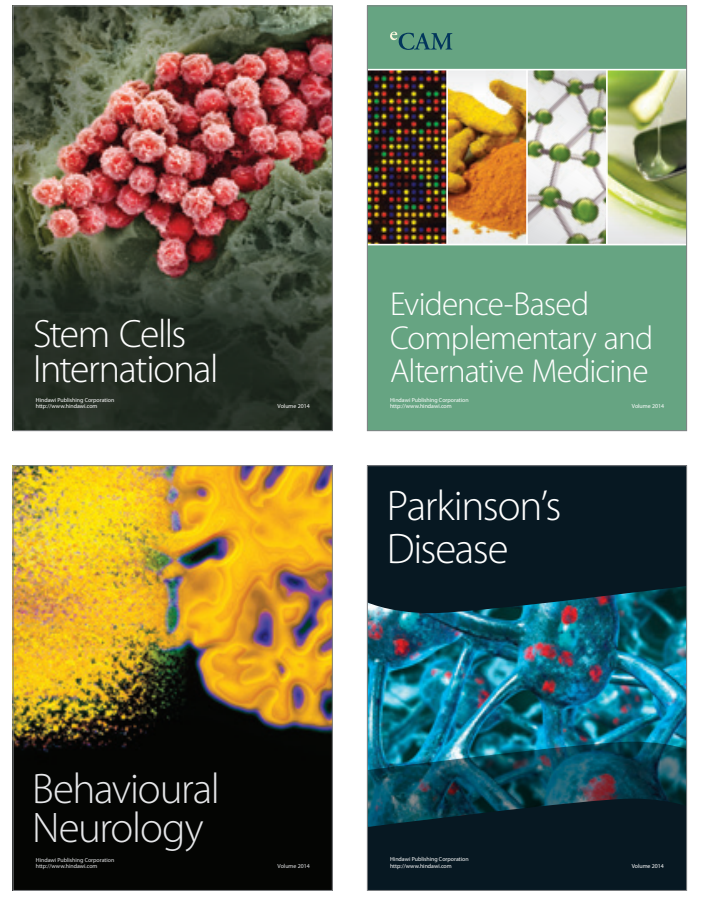
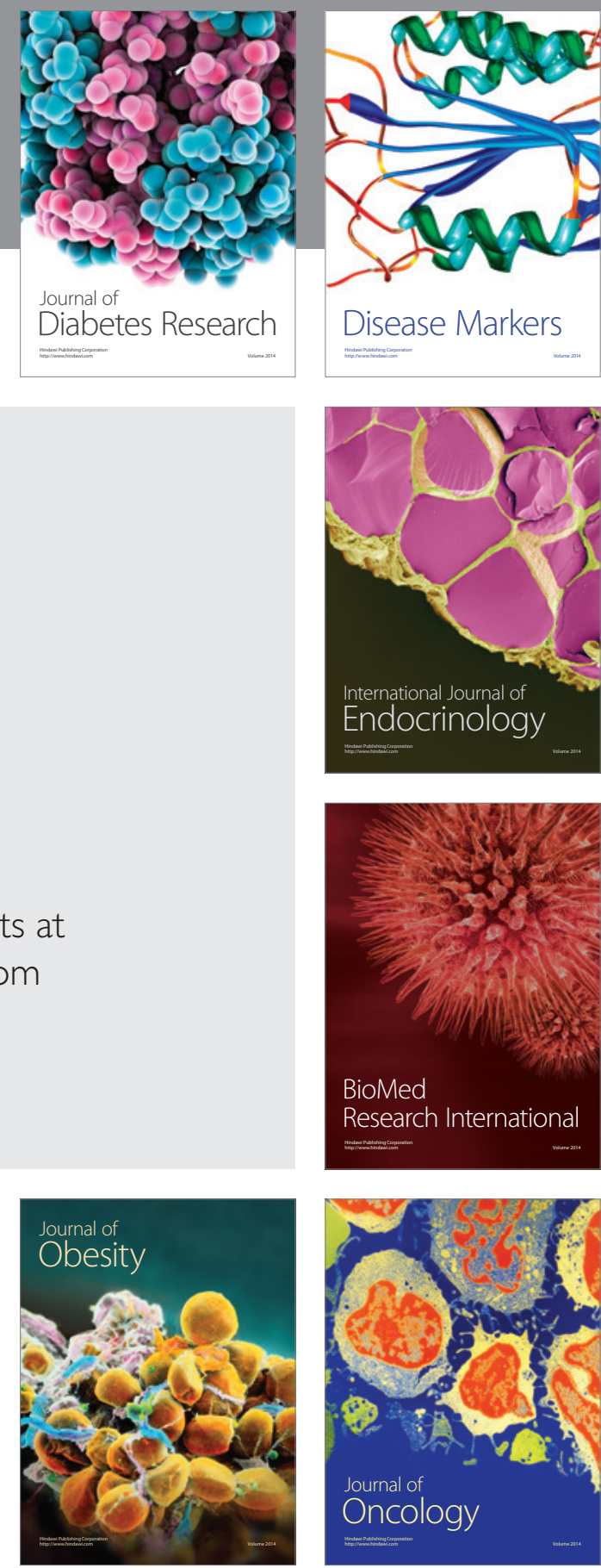

Disease Markers
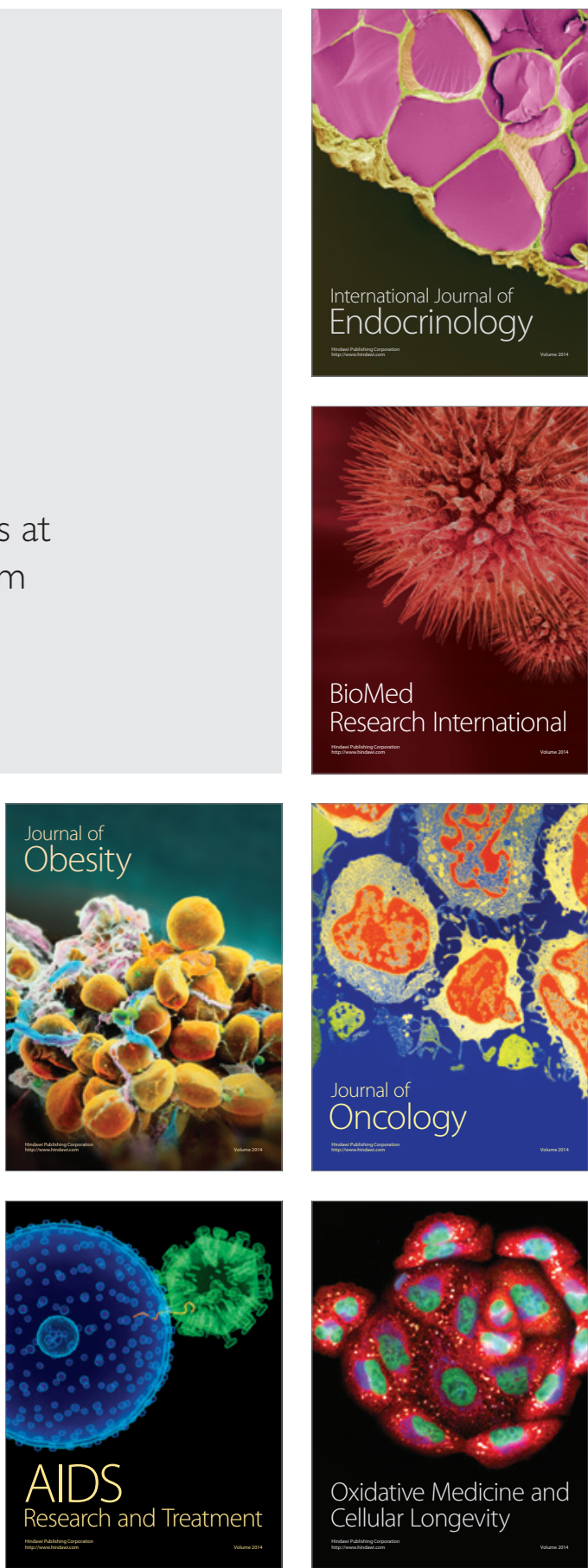